\title{
Internal biliary stenting during orthotopic liver transplantation: anastomotic complications, post-transplant biliary interventions, and survival
}

\begin{abstract}
Mathur AK, Nadig SN, Kingman S, Lee D, Kinkade K, Sonnenday $\mathrm{CJ}$, Welling TH. Internal biliary stenting during orthotopic liver transplantation: anastomotic complications, post-transplant biliary interventions, and survival.
\end{abstract}

Abstract: Background: Biliary complications are a leading source of surgical morbidity following orthotopic liver transplantation (OLT). Methods: We examined how prophylactic internal biliary stent placement during OLT affected post-transplant morbidity and mortality in a single-center retrospective cohort study of 513 recipients (20062012). Recipient and donor covariates were collected. Biliary complications included major and minor anastomotic leaks, strictures, or stenoses. Multivariate regression models were created to estimate how operative biliary stents affected outcomes.

Results: About $87.3 \%(\mathrm{n}=448)$ of recipients had a duct-to-duct biliary anastomosis, and $43.1 \%(\mathrm{n}=221)$ had biliary stents placed. The biliary complication rate was $<15 \%$ at five $\mathrm{yr}$, and $44.8 \%(\mathrm{n}=230)$ overall. Stenting was not protective from anastomotic biliary complications $(p=0.06)$. Stenting was associated with a $74 \%$ higher adjusted risk of needing multiple endoscopic retrograde cholangiographies (ERCs; odds ratio $[\mathrm{OR}] 1.74, \mathrm{p}=0.011$ ), and trended toward a lower adjusted risk for repetitive percutaneous transhepatic cholangiography (PTCs; OR 0.56, $\mathrm{p}=0.063)$. Stenting had no effect on the cumulative freedom from biliary complications $(\mathrm{p}=0.94)$. Biliary complications were associated with mortality (HR 1.86, $\mathrm{p}=0.014)$ and was unaffected by stenting $(\mathrm{aHR}=0.72, \mathrm{p}=0.246)$.

Conclusions: Biliary stenting during OLT does not deter biliary complications and is associated with higher risk of multiple invasive biliary interventions, particularly ERCs. Surgeons should evaluate the utility of biliary stents at OLT within this context.

\author{
Amit K. Mathur ${ }^{a, b}$, Satish N. \\ Nadig $^{c}$, Stephanie Kingman ${ }^{d}$, \\ Dustin Lee $^{\mathrm{e}}$, Kathleen Kinkade ${ }^{\mathrm{e}}$, \\ Christopher J. Sonnenday ${ }^{e}$ and \\ Theodore H. Welling ${ }^{e}$
}

aDivision of Transplant Surgery, Department o Surgery, Mayo Clinic Arizona, Phoenix, AZ, ${ }^{b}$ Mayo Clinic Robert D. and Patricia E. Kern Center for Science of Health Care Delivery, 'Division of Transplant Surgery, Department of Surgery, Medical University of South Carolina, Charleston, SC, 'Department of Surgery, University of California at Los Angeles, Los Angeles, CA and esection of Transplantation Surgery, Department of Surgery, University of Michigan, Ann Arbor, MI, USA

Key words: bile duct - biliary complications liver transplant - stenting

Corresponding author: Amit K. Mathur, MD, Division of Transplant Surgery, Department of Surgery, Mayo Clinic Arizona, 5777 E Mayo Blvd, Phoenix, AZ 85054, USA.

Tel.: +1 480342 1010;

fax: +1 480342 2324;

e-mail: mathur.amit@mayo.edu

Conflict of interest: None.

Accepted for publication 15 January 2015
Despite significant advances in orthotopic liver transplantation (OLT) technique, and recipient and donor selection, complications arising from the biliary anastomosis are a continued source of significant morbidity and mortality for patients after transplant. Recent reports identify a significant rate of these complications, up to $30-40 \%$ over time (1-5). Biliary complications significantly increase morbidity for patients and are costly for hospitals (6). Several risk factors have been associated with a higher risk of biliary complications including the use of partial liver grafts, cold and warm ischemic time, arterial complications, donor age, weight, graft steatosis, and recipient smoking status (3, 5-8). Many transplant surgeons have looked to stenting the biliary anastomosis using internal silastic pediatric feeding tubes, transcystic tubes, or T-tubes to preclude these complications.

T-tubes have not demonstrated a clear benefit in prospective trials, but other biliary stenting techniques have shown promise in retrospective studies $(4,5,9)$. The use of operatively placed internal biliary stents remains controversial, underscoring the wide variation in the surgical management of the bile duct in liver transplantation (10). It is further 
unknown whether intra-operative internal biliary stents themselves contribute to any post-OLT morbidity. Depending on the type of stent used, various complications may occur. Cholangitis due to stent obstruction, intrahepatic stent migration, hemobilia, visceral injury, intestinal fistula, or biliary peritonitis after stent removal have been reported $(9,11-20)$. These complications may require additional invasive treatments. Endoscopic retrograde cholangiography (ERC) stents and percutaneous transhepatic cholangiographic (PTC) catheterization are the treatments of choice for anastomotic biliary leaks and strictures after OLT, but are costly, invasive, and carry their own risks. It is unknown how the practice of intra-operative internal biliary stenting affects the need for these interventions after OLT.

In this study, we retrospectively reviewed our experience with intra-operative internal biliary stent placement during OLT, subsequent biliary complications, and utilization rates of invasive biliary interventions, including ERC, PTC, and esophago-gastric-duodenoscopy (upper endoscopy) (EGD). We further evaluated the effects of stenting practices on post-OLT survival to better inform surgeons about the risks and benefits of this practice and improve surgical decision-making.

\section{Methods}

A total of 537 adult patients were retrospectively reviewed at our institution from January 1, 2006, to December 31, 2012. Recipient and donor demographics, transplant procedural factors, and posttransplant events were collected from patient records. Recipient factors included age, sex, race/ ethnicity, primary diagnosis, Model for End-Stage Liver Disease (MELD) score, cardiovascular disease, hypertension, diabetes, and smoking status (active, former, lifetime non-smoker). Donor factors included age, sex, race/ethnicity, and donor type (brain death, donation after cardiac death, living donor). Procedural factors included type of graft (whole organ or split graft), primary transplant vs. retransplant, use of an internal biliary stent, cold ischemia time, warm ischemia time, and type of anastomosis.

\section{Surgical management and immunosuppression}

At transplant, the biliary anastomosis was constructed at the discretion of the attending surgeon based on anatomy and primary disease process and was either a choledochodochostomy or biliary-enteric anastomosis using a Roux-en-Y configuration. All biliary anastomoses were performed using $5-0$ or $6-0$ polydioxanone suture (PDS) in either a running or interrupted fashion. The donor and recipient bile ducts were also shortened and spatulated at the surgeon's discretion. The use of an internal biliary stent was also placed at the discretion of the surgeon performing the procedure and consisted of placing a silastic $5 \mathrm{~F}$ or $8 \mathrm{~F}$ pediatric feeding tube with customized side holes placed into the biliary tree and positioned with one end through the ampulla and the other end above the level of the anastomosis. Post-OLT immunosuppression included triple immunosuppressive therapy, which included a calcineurin inhibitor, mycophenylate mofetil, as well as steroids. Patients with renal dysfunction underwent induction immunosuppression with anti-CD25 chimeric monoclonal antibody therapy (basiliximab) with delayed initiation of calcineurin inhibitors. Patients with stents were verified to have passed their stents with use of plain abdominal radiography at three wk. Patients who failed to pass their stents spontaneously underwent elective EGD for stent removal.

\section{Defining biliary complications}

Biliary complications were identified by review of patient records and were diagnosed clinically (presence of biliary ascites, bile in drain, bilious wound drainage) or by imaging (ERC or PTC). Non-anastomotic or intrahepatic strictures were excluded from this study $(n=24)$, which were identified based on appearance on cholangiogram. The decision to perform either ERC or PTC was based on any suspicion of a biliary complication based on clinical judgment and laboratory studies. All biliary complications that occurred following liver transplantation were included in the analysis until the end of follow-up. Biliary leaks were defined as major or minor, as determined by clinical interpretation of cholangiogram studies by surgeons, radiologists, and gastroenterologists. The incidence of an invasive biliary intervention including ERC, PTC, or EGD was also recorded for each patient, and counts of each procedure per patient were recorded. Notably, our center practice utilizes ERC as a first-line intervention for suspected biliary complications in patients with choledochocholedochostomy. PTC was reserved for those patients with suspected leaks or strictures at a biliaryenteric anastomosis, or with any severe leak to facilitate internal and external biliary drainage.

\section{Statistical analysis}

Our primary exposure variable was the use of intra-operative biliary stents at OLT. We classified 
and calculated differences in the rates of specific biliary complications using univariate statistics. The Kaplan-Meier method was used to estimate biliary complication event rates over time. To determine the effect of stenting on biliary complications, we used a similar multivariate Cox model approach to determine the time to first biliary complication as we have previously described (5). The adjustment covariates included the recipient, donor, and transplant procedural factors, and year of transplant. The effect of hepatic artery thrombosis was also included in the model as a time-dependent covariate. Each model was censored at death or at the end of follow-up. Covariates were included in the model based on a significant effect on univariate analysis or on potential biological plausibility.

We further designed models to determine whether stenting was associated with repetitive invasive biliary intervention. We used univariate and multivariate ordinal logistic regression analyses to identify whether the biliary stenting affected the occurrence of multiple ERCs, PTC interrogations, and EGDs. Ordinal logistic regression measures the effect of an exposure covariate on the rate of transition in an ordered response variable; that is, the occurrence of a third ERC happens only after a second ERC. This model structure is parsimonious and decreases any bias that may arise from using a count data model structure, which assumes outcomes as a part of a stochastic process. To evaluate longer-term outcomes, we used multivariate Cox regression methods and the NelsonAalen hazard estimator to evaluate differences in the rate of death-censored freedom from biliary complications based on stent utilization. These models were adjusted for recipient and donor demographics, clinical factors including diagnosis, recipient comorbidities, laboratory MELD at transplant, and year of transplant. We additionally designed multivariate Cox proportional hazards regression models to determine whether biliary complications had any effect on survival after liver transplant, and whether this was modified by the use of a stent in the odds ratio (OR). These models were censored for occurrence of retransplant. Risk adjustment covariates included recipient and donor demographics, clinical factors including diagnosis, recipient comorbidities, laboratory MELD at transplant, year, cold ischemic time, and warm ischemic time.

The study was approved by the local Institutional Review Board. STATA version 10.0 (College Station, TX, USA) was used to complete all statistical analyses. Statistical significance was defined at a $p$ value of $<0.05$.

\section{Results}

After exclusions for the development of intrahepatic or non-anastomotic strictures $(\mathrm{n}=24), 513$ patients of the study cohort were included in the final analysis. Two hundred and twenty-one patients $(43 \%)$ were stented internally in the operating room at the time of liver transplant. By year, there was a significantly greater utilization of stents with time, ranging from $19.8 \%$ of transplants in 2006 to $63.8 \%$ in 2012 . The clinical characteristics of the study population are shown in Table 1. There were some recipient-, donor-, and operation-specific differences between the stented and non-stented population on univariate analysis. Female patients $(\mathrm{n}=248)$ made up $48 \%$ of the study cohort. The majority of patients in each racial/ethnic group were not stented. Female patients had almost a threefold higher odds of not being stented than being stented (OR 2.70, $\mathrm{p}<0.001)$. Hepatocellular cancer recipients were overrepresented in the stent group (stented 32.1\% vs. non-stented $26.7 \%$ ), likely owing to the concomitant era effects in stent usage and shift toward more transplants for this diagnosis. MELD scores at transplant were similarly distributed between stent and non-stent groups. Seventy-one percentage of retransplants were not stented. Greater than $90 \%$ of patients in each group received liver grafts from brain-dead donors. The distribution of donor causes of death was similar between stent and nonstent groups, but death from cerebrovascular causes was almost twofold higher in the stent population. From a technical standpoint, warm ischemia time was lower in the stent group, likely related to secular changes in operative technique over time. The rates of choledochodochostomy were similar between stent and non-stent groups ( $48 \%$ vs. $52 \%$, respectively), but over $90 \%$ of patients who had a biliary-enteric anastomosis were not stented.

Within the first five yr after transplant, $<15 \%$ of all patients had a biliary diagnosed complication. However, biliary complications did affect $44.3 \%$ of patients in long-term follow-up (Fig. 1). By era, the complication rate declined, with $31 \%$ of recipients presenting with biliary complications in 2012 at the end of follow-up. Of those who had a biliary complication $(\mathrm{n}=230), 46.1 \%$ had anastomotic leaks $(\mathrm{n}=109)$, but only $25 \%$ of those were classified as major leaks $(n=27,5 \%$ of total population). By stent group, a greater proportion of patients with bile leaks were not stented, but stented patients had a higher rate of biliary strictures and leaks with a subsequent stricture. Overall, there was no significant association between rate of 
Mathur et al.

Table 1. Clinical and demographic differences between 513 liver transplant recipients by intra-operative internal biliary stenting practice

\begin{tabular}{|c|c|c|c|}
\hline Variable & No biliary stent $(n=292)$ & Internal biliary stent $(n=221)$ & p Value \\
\hline \multicolumn{4}{|l|}{ Recipient factors } \\
\hline Age at transplant (yr) & $52.3 \pm 10.3$ & $52.6 \pm 9.7$ & 0.71 \\
\hline Sex (\% female) & $181(62)$ & $67(30.3)$ & $<0.001$ \\
\hline Race (\% group) & & & 0.15 \\
\hline White & $240(82.2)$ & $172(77.8)$ & \\
\hline Black & $29(9.9)$ & $26(11.8)$ & \\
\hline Asian & $10(3.4)$ & $4(1.8)$ & \\
\hline Other & $13(4.5)$ & $19(8.6)$ & \\
\hline Median Model for End Stage Liver & $21(16,24)$ & $20(14,27)$ & 0.41 \\
\hline \multicolumn{4}{|l|}{ Disease score (IQR) } \\
\hline Primary diagnosis (\%) & & & $<0.001$ \\
\hline Hepatitis B/C $(n=104)$ & $57(19.5)$ & $47(20.3)$ & \\
\hline EtOH cirrhosis $(n=58)$ & $27(9.3)$ & $31(14.0)$ & \\
\hline $\operatorname{HCC}(n=149)$ & $78(26.7)$ & $71(32.1)$ & \\
\hline Cholestatic $(n=77)$ & $65(22.3$ & $12(5.4)$ & \\
\hline Cryptogenic $(n=55)$ & $23(7.8)$ & $32(11.5)$ & \\
\hline Fulminant $(n=14)$ & $6(2.1)$ & $8(3.6)$ & \\
\hline Other $(n=37)$ & $22(7.5)$ & $15(6.8)$ & \\
\hline Retransplant $(n=42)$ & $30(10.3)$ & $12(5.4)$ & \\
\hline \multicolumn{4}{|l|}{ Comorbidities (\%) } \\
\hline Hypertension $(n=159)$ & $93(31.9)$ & 66 (29.9) & 0.63 \\
\hline Diabetes mellitus $(n=136)$ & $83(28.4)$ & $53(24.0)$ & 0.26 \\
\hline Coronary artery disease $(n=34)$ & $19(6.5)$ & $15(6.8)$ & 0.90 \\
\hline Smoking (\%) & & & 0.28 \\
\hline Lifetime non-smoker $(n=253)$ & $150(51.4)$ & $103(46.6)$ & \\
\hline Former smoker $(n=217)$ & $115(39.4)$ & $102(46.1)$ & \\
\hline Active smoker $(n=43)$ & $27(9.3)$ & $16(7.3)$ & \\
\hline \multicolumn{4}{|l|}{ Donor factors } \\
\hline Age at donation (yr) & $38.3 \pm 16.5$ & $39.3 \pm 16.2$ & 0.48 \\
\hline Sex (\% female) & $110(39.9)$ & $83(39.0)$ & 0.84 \\
\hline \multicolumn{4}{|l|}{ Cause of death (\%) } \\
\hline Anoxia $(n=193)$ & $114(39.0)$ & $79(35.8)$ & 0.05 \\
\hline Cerebrovascular $(n=68)$ & $28(9.6)$ & $40(18.1)$ & \\
\hline Cardiovascular $(n=25)$ & $17(5.8)$ & $8(3.6)$ & \\
\hline Head trauma $(n=182)$ & $104(35.6)$ & $78(35.3)$ & \\
\hline Living donor $(n=3)$ & $1(0.34)$ & $2(0.90)$ & \\
\hline Other $(n=45)$ & $28(9.6)$ & $13(5.9)$ & \\
\hline Donation after cardiac death $(n=26)$ & $13(4.5)$ & $13(5.9)$ & 0.47 \\
\hline \multicolumn{4}{|l|}{ Transplant factors } \\
\hline Split graft (\%) & $11(3.8)$ & $12(5.4)$ & 0.36 \\
\hline Cold ischemia time (min) & $424.5 \pm 139.4$ & $402.2 \pm 174.5$ & 0.11 \\
\hline Warm ischemia time (min) & $34.3 \pm 14.3$ & $30.2 \pm 8.1$ & 0.0002 \\
\hline Biliary anastomosis type (\%) & & & $<0.001$ \\
\hline Choledochodochostomy $(n=448)$ & $233(79.8)$ & $215(97.3)$ & \\
\hline Roux-en-Y hepaticojejunostomy $(n=65)$ & $59(20.2)$ & $6(2.7)$ & \\
\hline
\end{tabular}

biliary complication and stent usage on univariate analysis $(\mathrm{p}=0.06)$.

Fig. 2 demonstrates the distribution of invasive procedures utilized for biliary complications based on stent usage. Across the entire population, 2062 interventions were performed in the cohort. Patients averaged $4.1 \pm 8.1$ invasive procedures in total. There were significantly more procedures performed in patients who were not stented (nonstent vs. stent, 1291 vs. 771, p < 0.05), owing to the significantly greater number of PTC placement and exchanges in patients who were not stented. There was no difference in the utilization of ERC for cause in the stented population (non-stent vs. stent, 339 vs. $358, \mathrm{p}=\mathrm{NS}$ ). Interestingly, there were slightly greater number of EGD procedures performed for stent retrieval in the group who were not stented in the OR, likely owing to the subsequent need for an ERC-placed endo-biliary stent and an obligatory procedure for stent retrieval at a later point.

The association between the use of an intraoperative stent and the likelihood of repetitive biliary intervention is depicted in Fig. 3. Intraoperative biliary stenting was associated with a twofold higher odds of requiring multiple ERCs 
Fig. 1. Rates of specific biliary complications after orthotopic liver transplantation (OLT) by intraoperative biliary stent use, $\mathrm{n}=513$. About $43.1 \%$ of patients underwent intra-operative biliary stenting at OLT, but there was no significant difference in the distribution or type of biliary complications identified postoperatively based on the stent utilization. There was a trend toward more leaks in the non-stented group, and more strictures in the stented group, but these were not statistically significant on univariate analysis.
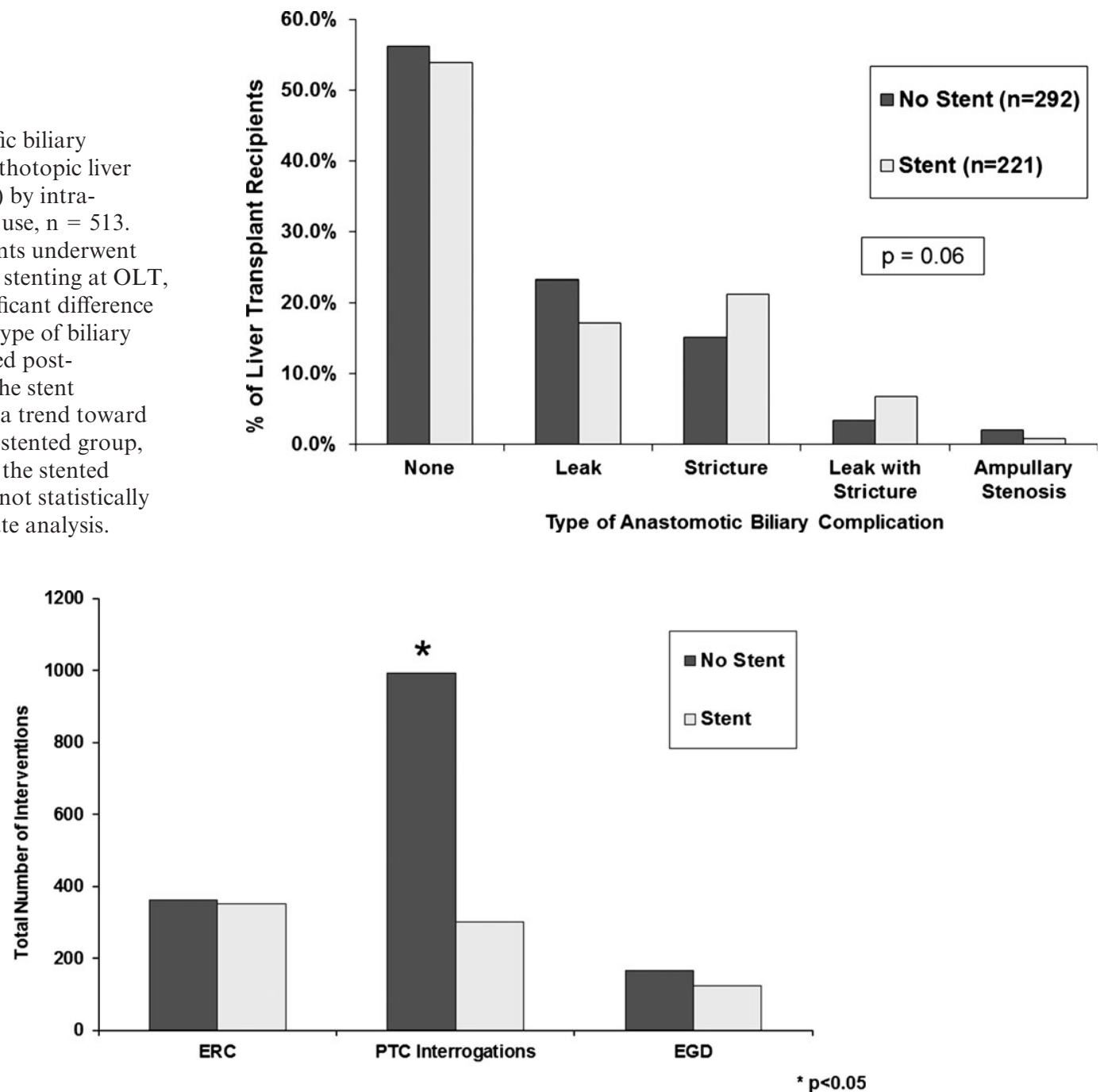

Fig. 2. Total number of biliary interventions after orthotopic liver transplantation (OLT) by intra-operative biliary stent use. A total of 2062 procedures were performed across the OLT population $(\mathrm{n}=513)$, averaging four invasive biliary procedures per patient. There were significantly more procedures performed in the non-stent group $(n=1291)$ vs. stent group $(n=771 ; p<0.05)$, which primarily was driven by differences in the total number of percutaneous transhepatic cholangiography (PTC) procedures performed in non-stented patients. These differences were unadjusted for other clinical covariates.

Fig. 3. Likelihood of repetitive invasive interventions for biliary complications based on utilization of an intra-operative biliary stent. The effect of intra-operative biliary stenting on utilization of different invasive biliary procedures was evaluated using unadjusted and adjusted multivariable ordinal logistic regression models. Even after adjusting for clinical covariates, stented patients had nearly twofold higher odds of multiple endoscopic retrograde cholangiographies (ERCs).

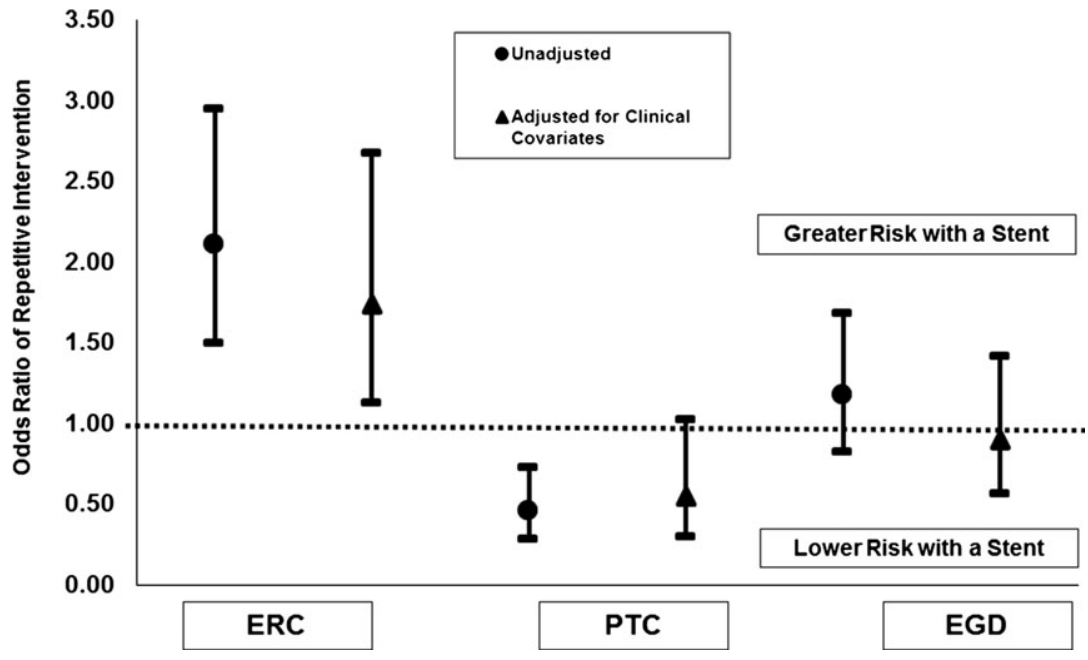


(OR 2.11, p < 0.001). After adjusting for clinical risk factors, there was a $74 \%$ higher risk of patients needing multiple ERCs associated with intra-operative biliary stenting. However, intra-operative stenting was associated with a $54 \%$ lower odds of requiring multiple PTC exchanges on unadjusted analysis (OR 0.46, $\mathrm{p}=0.001)$. The risk-adjusted model suggested a trend toward lower rates of repetitive PTCs, but this was not statistically significant (OR 0.56, $\mathrm{p}=0.06$ ). Over time, beyond the peri-transplant period, stented and non-stented patients had similar odds of upper endoscopy. However, in a subgroup analysis, within the first $90 \mathrm{~d}$, stented patients had a threefold higher odds of needing multiple upper endoscopy procedures (data not shown; OR 3.58, $\mathrm{p}=0.01$ ).

Figs. 4 and 5 demonstrate the long-term outcomes of patients in the study cohort. After the development of biliary complications, there was no significant difference in the rate of freedom from having an indwelling biliary stent or needing additional biliary therapy between those stented at OLT vs. those who were not on unadjusted and adjusted analysis $(\mathrm{p}=0.94)$. Notably, the presence of an anastomotic biliary complication was significantly associated with increased adjusted risk of mortality after OLT $(\mathrm{HR}=1.87, \mathrm{p}=0.01$; Fig. 5), and intra-operative internal stenting was not associated with any effect on survival (HR 0.71, $\mathrm{p}=0.246)$.

\section{Discussion}

Anastomotic biliary complications are the Achilles' heel of the liver transplant operation. Rates of biliary complications after liver transplant vary across the literature $(1,2,21)$ and are reported to affect as many as $10-40 \%$ patients after transplant $(5,6)$. Several authors have described the incidence, treatment, and management of biliary complications after deceased donor and living donor liver transplant $(1,2,4-6,21)$. A salient patient-centered outcome in liver transplantation is the number and invasiveness of procedures used to manage biliary complications. While the management of these complications has been driven by non-operative endoscopic and percutaneous advances, these procedures are inconvenient, uncomfortable, and carry quantifiable risks of major complications (12). While the use of T-tubes has been studied, it is unknown whether intra-operative internal biliary stenting affects the utilization of these procedures, which we measured by the number and the need for repetitive invasive interventions. Stenting the biliary tree using internal feeding tubes at transplant, in this cohort, did not reduce the risk of biliary complications or mortality and was associated with greater morbidity. Stented patients had higher adjusted risk of needing multiple ERCs over time and EGDs in the first $90 \mathrm{~d}$, without any protection in the rate of resolution of biliary complications. Non-stented patients had a significantly higher total number of PTC exchanges, but stent usage was not associated with any difference in the riskadjusted odds of repetitive PTC exchanges.

An entire body of transplant literature is devoted to biliary complications after liver transplant, which remain common and ultimately affect clinical outcomes significantly. In our population, there was a high rate of biliary complications. This likely has to do with our aggressive approach to diagnosing these problems and concern about pre-

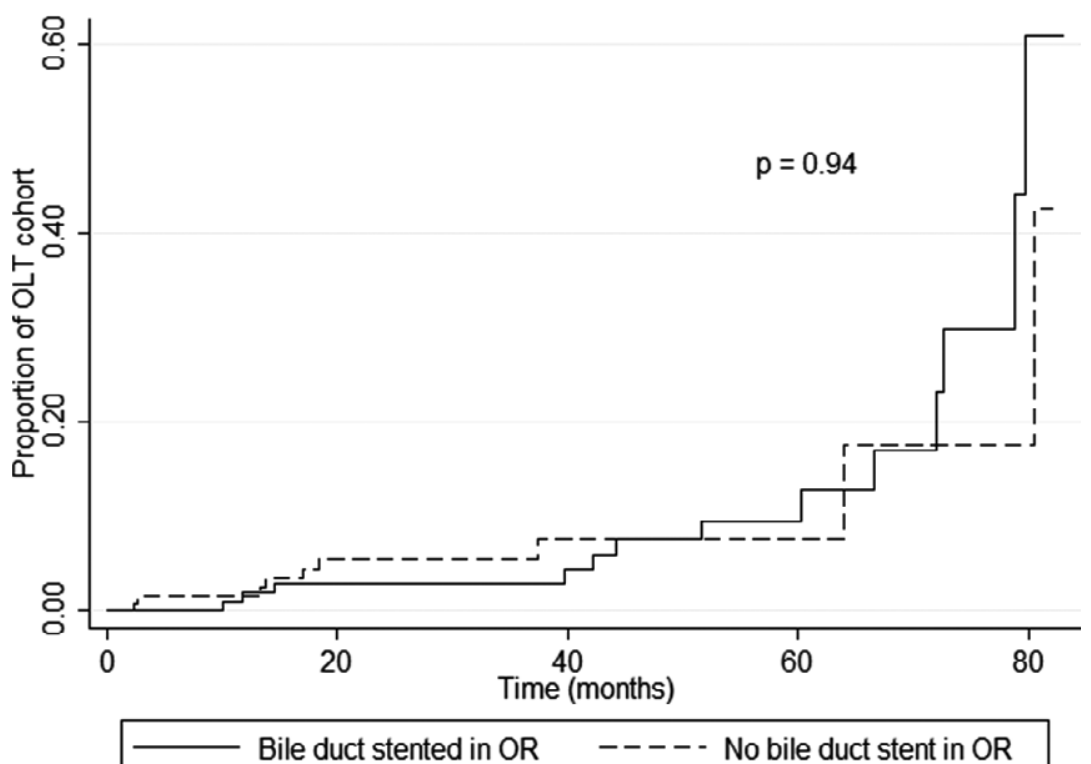

Fig. 4. Intra-operative biliary stenting at orthotopic liver transplantation (OLT) does not affect cumulative freedom from anastomotic biliary complications. Biliary complications were common in the study cohort, but intra-operative biliary stenting was not protective from the rate of freedom from biliary therapy, as defined by continued need for an in-dwelling stent in the bile duct or by the need for continued invasive biliary procedures. 
Fig. 5. Post-orthotopic liver transplantation (OLT) survival is significantly lower in patients with an anastomotic biliary complication. After liver transplant, anastomotic biliary complications were associated with significantly higher mortality on unadjusted and adjusted analyses. Intra-operative biliary stenting did not modify this association on multivariate analysis.

vent long-term biliary morbidity. This analysis represents the actual clinical experience in a large transplant program. An admitted limitation of the analysis is a bias toward identifying a complication, which may overestimate the complication rate, which is difficult to interpret retrospectively. Small leaks identified during an early ERC may in fact have been caused by high intraluminal pressure during contrast injection during the study. The rate of severe leaks among all patients with bile leaks was $25 \%$, and $10 \%$ of the study population had more than 10 PTC exchanges. This rate likely reflects local practice patterns at our institution in the long-term management of patients with complicated biliary pathology after transplant. After a thorough review of the data, the high number of PTC interrogations among non-stented patients is likely related to the experience of a few patients who had long-standing strictures that required multiple PTC treatments, some for months and years after transplant. These patients had complex strictures, some with associated arterial insufficiency, and were not candidates for ERCP based on recipient anatomy. These patients were essentially PTC tube dependent, were undergoing repetitive stent exchanges, and were not operative candidates for biliary revision or retransplantation. It is worth noting, however, that these few patients' large number of procedures did not skew the multivariate model designed to predict the need for multiple PTC procedures. After adjusting for clinical factors, stenting (or lack of stenting) was not associated with a risk of undergoing multiple PTC procedures for an individual patient.

No other studies have attempted to ascribe a stent-specific utilization rate for invasive biliary interventions after liver transplant. The reported complication rates after these procedures are low (22-24), but multiple procedures are needed when biliary complications are severe (15). In our cohort, liver transplant recipients averaged four invasive biliary procedures per patient, and we found significantly higher odds for ERC in stented patients, and threefold higher rates of EGD during the first three months after transplant. The higher rate of endoscopic interventions in the stented population was likely related to the need for stent retrieval secondary to stent occlusion, increased intraluminal debris, altered biliary chemistry, and reactive inflammation that may contribute to strictures that require multiple ERC treatments. Additionally, secondary complications from having a stent in place such as proximal stent migration, failure of stent passage, and cholangitis could also be responsible for the repetitive interventions. Severe biliary complications in choledochocholedochostomy or anastomotic biliary complications in patients with Roux-en-Y biliary-enteric drainage usually require PTC procedures with frequent exchanges. In our analysis, there was a trend toward lower rates of multiple PTC exchanges in the stented patients, but it is premature to state that stents are protective from severe complications or major leaks that usually require PTC procedures. The need for chronic in-dwelling PTCs with maintenance exchanges in this population is likely multifactorial and could be related to biliary complications in retransplant patients, the use of split liver grafts, hepatic artery complications affecting biliary perfusion, and poor surgical candidacy for operative biliary revision. With regard to survival, the overall survival of the cohort at one, three, and five $\mathrm{yr}$ is comparable to the rest of the literature and national data as well (25). It is 
therefore not surprising that placing a stent at transplant had no significant effect on mortality. The significant association between anastomotic biliary complications and death was more impressive, but death after liver transplant is often related to multiple factors, proximity to the operative event, accumulation of other organ dysfunction, and the clinical penetrance of comorbidities in the post-transplant course.

The independent effect of biliary complications on mortality was also notable, which has been shown elsewhere $(3,21,26)$, and importantly was not modified by biliary stents. This was particularly interesting, because the survival within the first post-transplant year was similar between those with and without biliary complications. This may be driven by an aggressive early approach to managing these complications by our group, with separation of the mortality curves over time due to a cascade of morbidity from these complications, including frequent hospitalization, chronic infection, debilitation, and attendant secondary complications.

While our study delineates the association between intra-operative biliary stent usage and the morbidity of multiple biliary interventions, there are limitations to our analysis. This study represents the experience of a single transplant program and the clinical biases therein. The choice to place biliary stents was based on prior clinical perceptions that internal biliary stents may avoid anastomotic leaks and limit the need for additional procedures. A clear era effect on stent usage was present as well, which we attempted to adjust for in our models. As a retrospective study, the clinical context and nuances related to medical decisionmaking in the management of post-transplant patients may be lost and therefore affect our findings. We excluded patients with intrahepatic biliary strictures, which we argue arise from an alternate mechanism. This exclusion may have also removed some patients who had anastomotic complications as well, which therefore may have underrepresented the biliary interventions needed specifically for those specific complications. These are limitations of many retrospective clinical studies, but this analysis shows an increased risk for repetitive biliary interventions in patients who were stented at transplant in an experienced liver transplant program.

The study clearly calls into question the utility of biliary stents in the operating room (6). This study and our experience have prompted us to eliminate biliary stent utilization at transplant and delay use of ERC early after transplant. Further study with a randomized controlled trial could help elucidate the true morbidity related to stents in subsets of patients deemed to be at higher risk. However, broader studies of surgical outcomes using granular multicenter clinical data may represent the best approach to feed directly back into quality improvement initiatives at transplant programs. Until such studies are initiated, liver transplant programs with an interest in complication management and surgical quality improvement will continue to rely on retrospective single-center studies from similar centers and analyses of their own experiences.

\section{References}

1. Gastaca M. Biliary complications after orthotopic liver transplantation: a review of incidence and risk factors. Transplant Proc 2012: 44: 1545.

2. Akamatsu N, Sugawara Y, Hashimoto D. Biliary reconstruction, its complications and management of biliary complications after adult liver transplantation: a systematic review of the incidence, risk factors and outcome. Transpl Int 2011: 24: 379.

3. Axelrod DA, Lentine KL, Xiao $H$ et al. National assessment of early biliary complications following liver transplantation: incidence and outcomes. Transplantation 2014: 98: 1226

4. Welling TH, Heidt DG, Englesbe MJ et al. Biliary complications following liver transplantation in the model for end-stage liver disease era: effect of donor, recipient, and technical factors. Liver Transpl 2008: 14: 73.

5. Mathur AK, Ranney DN, Patel SP et al. The effect of smoking on biliary complications following liver transplantation. Transpl Int 2011: 24: 58.

6. Englesbe MJ, Dimick J, Mathur A et al. Who pays for biliary complications following liver transplant? A business case for quality improvement. Am J Transplant 2006: 6: 2978.

7. Mathur AK, Heimbach J, Steffick DE, Sonnenday CJ, Goodrich NP, Merion RM. Donation after cardiac death liver transplantation: predictors of outcome. Am J Transplant 2010: 10: 2512.

8. Li HY, WeI YG, Li B et al. Impact of graft steatosis on the post-transplantation biliary complications for living donor liver transplant recipients in China. Hepatogastroenterology 2012: 59: 1194.

9. Gastaca M, Matarranz A, Munoz F et al. Biliary complications in orthotopic liver transplantation using choledochocholedochostomy with a T-tube. Transplant Proc 2012: 44: 1554.

10. Scatton O, Meunier B, Cherqui D et al. Randomized trial of choledochocholedochostomy with or without a $\mathrm{T}$ tube in orthotopic liver transplantation. Ann Surg 2001: 233: 432

11. Cerecedo-Rodriguez J, Phillips M, Figueroa-Barojas P et al. Self expandable metal stents for anastomotic stricture following liver transplant. Dig Dis Sci 2013: 58: 2661.

12. Giampalma E, Renzulli M, Mosconi C, Ercolani G, Pinna AD, Golfieri R. Outcome of post-liver transplant ischemic and nonischemic biliary stenoses treated with percutaneous interventions: the Bologna experience. Liver Transpl 2012: 18: 177.

13. Dumonceau JM, Tringali A, Blero D et al. Biliary stenting: indications, choice of stents and results: European 
Society of Gastrointestinal Endoscopy (ESGE) clinical guideline. Endoscopy 2012: 44: 277.

14. Depuydt P, Aerts R, Van Steenbergen W et al. An unusual case of rectal perforation after liver transplantation. Acta Chir Belg 2012: 112: 232.

15. Johnston TD, Reddy KS, Khan TT, Ranjan D. ERCP in the management of early versus late biliary leaks after liver transplantation. Int Surg 2006: 91: 301.

16. Tranchart H, Zalinski S, Sepulveda A et al. Removable intraductal stenting in duct-to-duct biliary reconstruction in liver transplantation. Transpl Int 2012: 25: 19.

17. Kusano T, Randall HB, Roberts JP, Ascher NL. The use of stents for duct-to-duct anastomoses of biliary reconstruction in orthotopic liver transplantation. Hepatogastroenterology 2005: 52: 695.

18. Pacholczyk M, Lagiewska B, Gontarczyk GW et al. Biliary complications following liver transplantation: single-center experience. Transplant Proc 2006: 38: 247.

19. Patkowski W, Nyckowski P, Zieniewicz K et al. Biliary tract complications following liver transplantation. Transplant Proc 2003: 35: 2316.

20. Losanoff JE, Vanha TG, Testa G, Ahmed EB, Millis JM. Endoscopic biliary stent migration to the iliopsoas muscle in a liver transplant recipient: percutaneous removal. Dig Dis Sci 2007: 52: 2508.

21. Zimmerman MA, Baker T, Goodrich NP et al. Development, management, and resolution of biliary complications after living and deceased donor liver trans- plantation: a report from the adult-to-adult living donor liver transplantation cohort study consortium. Liver Transpl 2013: 19: 259.

22. Ginat D, SaAd We, Davies MG, SaAd Ne, Waldman DL, Kitanosono T. Incidence of cholangitis and sepsis associated with percutaneous transhepatic biliary drain cholangiography and exchange: a comparison between liver transplant and native liver patients. AJR Am J Roentgenol 2011: 196: W73.

23. SAad WE, Davies MG, SAAd NE et al. Transhepatic dilation of anastomotic biliary strictures in liver transplant recipients with use of a combined cutting and conventional balloon protocol: technical safety and efficacy. J Vasc Interv Radiol 2006: 17: 837.

24. Elmunzer BJ, Debenedet AT, Volk ML et al. Clinical yield of diagnostic endoscopic retrograde cholangiopancreatography in orthotopic liver transplant recipients with suspected biliary complications. Liver Transpl 2012: 18: 1479.

25. OPTN/SRTR. 2011 Annual Data Report. Rockville, MD: Department of Health and Human Services, Health Resources and Services Administration, Healthcare Systems Bureau, Division of Transplantation, 2012.

26. Bustami RT, Ojo AO, Wolfe RA et al. Immunosuppression and the risk of post-transplant malignancy among cadaveric first kidney transplant recipients. Am J Transplant 2004: 4: 87. 